\title{
Second Generation Curvelet Transforms Vs Wavelet transforms and Canny Edge Detector for Edge Detection from WorldView-2 data
}

\author{
Mohamed Elhabiby $^{* a, b}$, Ahmed Elsharkawy ${ }^{\text {a,c }} \&$ Naser El-Sheimy ${ }^{\text {a,d }}$ \\ ${ }^{a}$ Dept. of Geomatics Engineering, University of Calgary, Calgary, Alberta, T2N 1N4 \\ Phone: 403-210-7897, Fax: 403-284-1980, \\ Email: mmelhabi@ucalgary.ca \\ ${ }^{b}$ Public Works Department, Faculty of Engineering, Ain Shams University, Cairo, \\ Egypt \\ c Email: askelsha@ucalgary.ca \\ d Email: elsheimy@ucalgary.ca
}

\begin{abstract}
Edge detection is an important assignment in image processing, as it is used as a primary tool for pattern recognition, image segmentation and scene analysis. Simply put, an edge detector is a high-pass filter that can be applied for extracting the edge points within an image. Edge detection in the spatial domain is accomplished through convolution with a set of directional derivative masks in this domain. On one hand, the popular edge detection spatial operators such as; Roberts, Sobel, Prewitt, and Laplacian are all defined on a 3 by 3 pattern grid, which is efficient and easy to apply. On the other hand, working in the frequency domain has many advantages, starting from introducing an alternative description to the spatial representation and providing more efficient and faster computational schemes with less sensitivity to noise through high filtering, de-noising and compression algorithms. Fourier transforms, wavelet and curvelet transform are among the most widely used frequency-domain edge detection from satellite images. However, the Fourier transform is global and poorly adapted to local singularities. Some of these draw backs are solved by the wavelet transforms especially for singularities detection and computation. In this paper, the relatively new multi-resolution technique, curvelet transform, is assessed and introduced to overcome the wavelet transform limitation in directionality and scaling.

In this research paper, the assessment of second generation curvelet transforms as an edge detection tool will be introduced and compared to traditional edge detectors such as wavelet transform and Canny Edge detector. Second generation curvelet transform provides optimally sparse representations of objects, which display smoothness except for discontinuity along the curve with bounded curvature. Preliminary results show the power of curvelet transform over the wavelet transform through the detection of nonvertical oriented edges, with detailed detection of curves and circular boundaries, such as non straight roads and shores. Conclusions and recommendations are given with respect to the suitability; accuracy and efficiency of the curvelet transform method compared to the other traditional methods
\end{abstract}

Keywords: High resolution satellite imagery, Edge detection, Canny operator, Curvelet transform, Wavelet transform, Building detection.

\section{Introduction}

One of the most important characteristics in an image is the features' edges, which can be described as a discontinuity in the local domain of the image. These discontinuities may result as gray, colors and texture variations [1]. Edge detection has broad applications in the domain of image processing, computer vision and so on. The influence of this process comes from the fact that it usually lies at the bottom of the classification process to serve as a base map for all other

DOI : $10.5121 /$ ijcses.2012.3401 
coming modules. Consequently, the more accurate this process is, the more accurate the whole classification results.

In this research paper an implementation of the second generation curvelet transform for edge detection will be introduced and a comparison with wavelet transform and the optimal edge detector operator, Canny, will be done.

Urban studies, coastal erosion, and agricultural surveys are a few examples where edge detection can be utilized. In the past few years, the development of edge detection techniques in the analysis of multi-temporal remote sensing imagery has been intensively growing. For many years, satellite based remote sensing has been a priceless tool for change detection. No other platform can constantly revisit an area, quantify and classify land cover or land use on such a broad scale. Satellite imagery is proving to be a cost-effective alternative to aerial photography, especially, for the acquisition of Land Cover information[2].

Second generation curvelet transform provides optimally sparse representations of objects, which display smoothness except for discontinuity along the curve with bounded curvature [3]. Some papers have investigated this technique for edge detection in high-resolution satellite imagery such as IKONOS or QuickBird, and microscopic imagery, $[1,4-6]$ which show a great potential of using curvelet transform in solving edge detection problems.

In the following sections, a brief introduction about implementation of the three methods in edge detection will be introduced followed by Data used description and methodology section, then the results and analysis section and finally, the Conclusions.

\section{Edge detection techniques}

\subsection{Canny edge detector}

Canny edge detection is an optimal method for step edges detection. Canny used three criteria to design his edge detector.

- Reliable detection of edges with low probability of missing true edges, and a low probability of detecting false edges.

- The detected edges should have a minimum distance to the true location of the edge.

- There should be only one response to a single edge (thin lines for edges).

Based on these criteria, the Canny edge detector first smoothes the image to eliminate any noise, then it finds the image gradient to highlight regions with high derivatives. The regions with high derivatives are tracked by the algorithm to suppress any pixel that is not at the maximum (non-maximum suppression). Two thresholds $T_{1}$ and $T_{2}$ are then introduced. If the magnitude of certain pixel is below $T_{1}$, it is set to zero (none edge), if the magnitude is above $T_{2}$, it is made an edge. And if the magnitude is between the two thresholds, then it is set to zero unless there is a path from this pixel to a pixel with a gradient above $T_{2}$ [7].

\subsection{Wavelet and edge detection}

Multi-resolution techniques aim at transforming images into a representation where both spatial and frequency information can be identified [8]. A wavelet transform decomposes images into a complete set of wavelet functions, which then form a basis, generally orthogonal. These functions are constructed by translating and dilating a single-mother wavelet which is localized in both spatial and frequency domain [9]. Once this is completed in discrete steps, the discrete wavelet transform is obtained, for which there exists an efficient filtering implementation in the real space. Every wavelet corresponds to a high and low-pass filter. For the most common cases with dilations by a factor of two, the scheme is called "dyadic" wavelet transform [8]. 


\subsection{Curvelet transform}

Initial introduction of Curvelet transforms technique was originally introduced by Candes and Donoho in 1999 as a result of the increasing demand of the presence of effective multiresolution analysis that has the ability to overcome the drawbacks of wavelet analysis. The transform was designed to represent edges and other singularities along curves much more efficiently than traditional transforms, i.e. using significantly fewer coefficients for a given accuracy of reconstruction [10]. Later and based on a frequency partition technique, the same authors proposed a considerably simpler second-generation curvelet transform. This secondgeneration curvelet transforms is meant to be easier to understand and use. It is also faster and less redundant compared to its first generation version [11]. In the new version of curvelet, the ridgelet transforms was discarded, thus reducing the amount of redundancy in the transform and increasing the speed considerably. Curvelet transforms is defined in both continuous and digital domain. Moreover, it can be used for multi-dimensional signals. Since the image-based feature extraction requires only 2D FDCT, Fast Discrete Curvelet Transforms, the discussion will be focused on purely two-dimensional applications and implementations [3].

\subsection{Discrete Curvelet Transform}

Coronae and rotations, as in the continuous-time definition, are not especially adapted to Cartesian arrays, so it is convenient to replace these concepts by Cartesian equivalents; here, "Cartesian coronae" based on concentric squares (instead of circles) and shears.
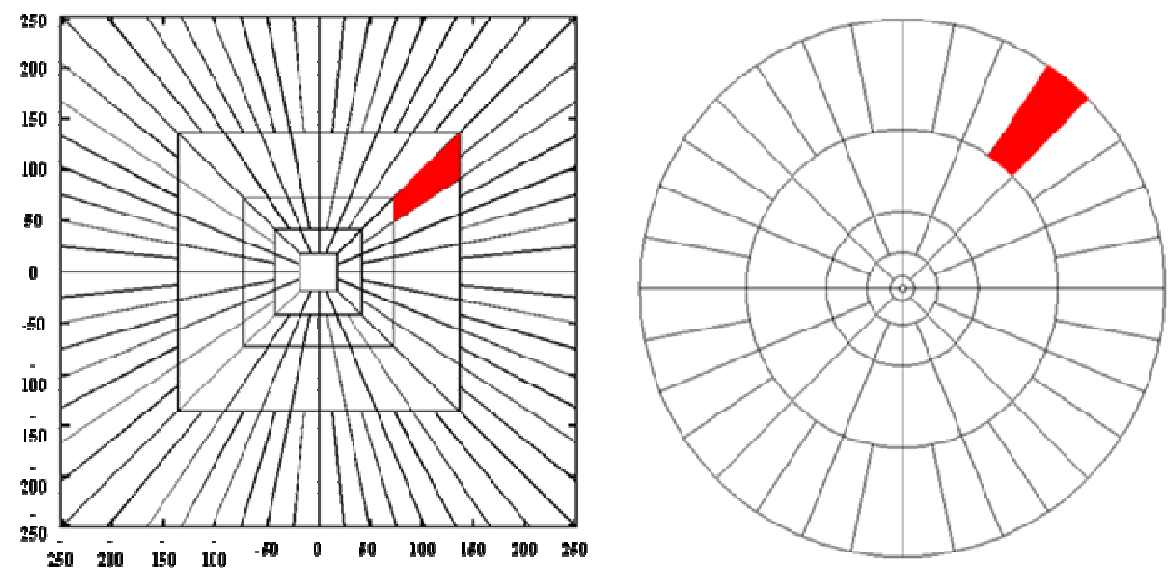

Figure 1 The transition from the continuous-time definition (right) to the discrete-time definition(left) [3].

Figure 1 (right) illustrates the basic digital tiling where the windows ' $U_{\mathrm{j}}$ ' smoothly localize the Fourier transform near the sheared wedges obeying the parabolic scaling. The shaded region represents one such typical wedge.

Now the Cartesian window $\widetilde{\mathrm{U}}$ is defined as:

$$
\hat{U}_{j, l}(\omega):=\hat{V}_{j}(\omega) \dot{V}_{j}\left(S_{A l} \omega\right) \quad \text { Eq.1 }
$$

Where: 
International Journal of Computer Science \& Engineering Survey (IJCSES) Vol.3, No.4, August 2012

$$
\left\{\begin{array}{l}
\tilde{W}_{j}(\omega)-\sqrt{\phi_{j+1}^{2}(\omega)-\phi_{j}^{2}(\omega)}, \\
\tilde{V}_{j}(\omega)=v\left(\frac{2^{\left\lfloor\frac{i}{2}\right\rfloor} \omega_{2}}{\omega_{1}}\right)
\end{array}\right.
$$

$\Phi$ is defined as the product of low-pass one dimensional windows:

$$
\phi_{j}\left(\omega_{1}, \omega_{\eta}\right)=\phi\left(2^{-\jmath} \omega_{1}\right) \phi\left(2^{-\jmath} \omega_{2}\right) \quad \text { Eq. } 2
$$

And $S_{\square}$ is the shear matrix:

$$
S_{\theta}=\left(\begin{array}{cc}
1 & 0 \\
-\tan \theta & 1
\end{array}\right)
$$

Hence, the discrete curvelet coefficients are defined as:

$$
C(j, l, k):=\int \hat{f}(\omega) \widetilde{U}_{j}\left(S_{\theta l}^{-1} \omega\right) e^{i\left(S_{\theta l}^{-l} b, \omega\right)} d \omega \quad \text { Eq.3 }
$$

According to [3], there are two different digital implementations of FDCT:

- Curvelets via USFFT (Unequally Spaced Fast Fourier Transform)

- And Curvelets via Wrapping.

The values of curvelet coefficients depend on how they are aligned in the real image. One can expect higher coefficients' values when the curvelet is accurately aligned to a given curve in an image. A very clear explanation is provided in figure 4 . The curvelet named ' $c$ ' in the figure is almost perfectly aligned with the curved edge and therefore, has a higher coefficient value. Curvelets ' $a$ ' and ' $b$ ' will have coefficients close to zero as they are quite far from alignment with the curve [5].

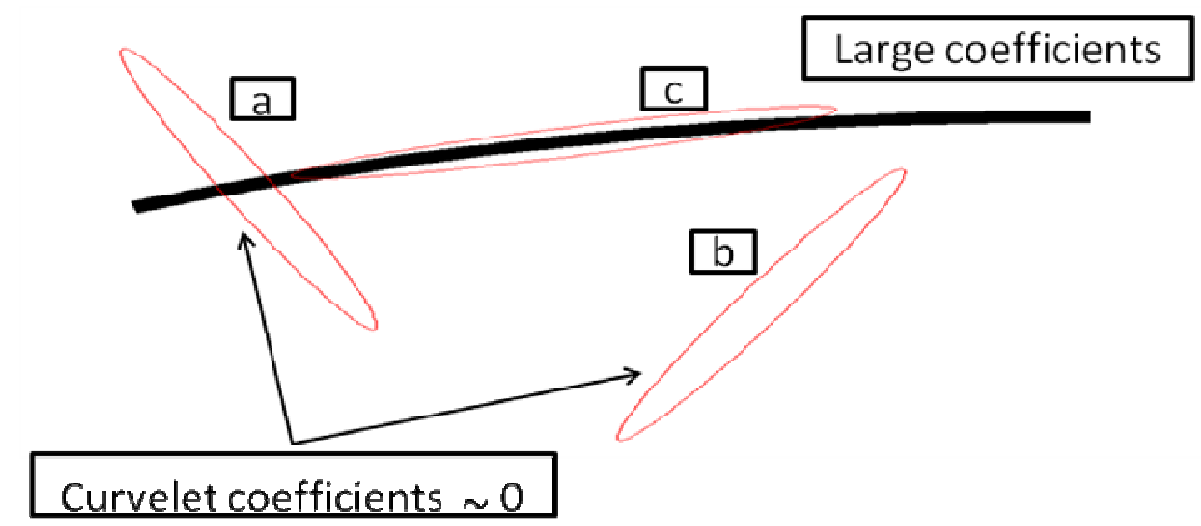

Figure 4 Alignment of curvelets along curved edges [5]

The unique mathematical property for representing curved singularities in a non-adaptive manner makes the Curvelet transform a higher-dimensional generalization of wavelets. 
The major advantage of the curvelet transforms compared to the wavelet is that the edge discontinuity is better approximated by curvelets than wavelets. Curvelets can provide solutions to the limitations that are apparent in wavelet transform and summarized as follows:

- Curved singularity representation,

- Limited orientation (Vertical, Horizontal and Diagonal)

- And absence of anisotropic element (isotropic scaling)

Figure 5 shows the edge representation capability of wavelet (right) and curvelet transform (left). More wavelets are required for an edge representation using the square shape of wavelets at each scale, compared to the number of required curvelets, which are of an elongated needle shape. The main idea here is that the edge discontinuity is better approximated by curvelets than wavelets. Curvelets can provide solutions for the limitations (curved singularity representation, limited orientation and absence of anisotropic element) existing in the wavelet transform.
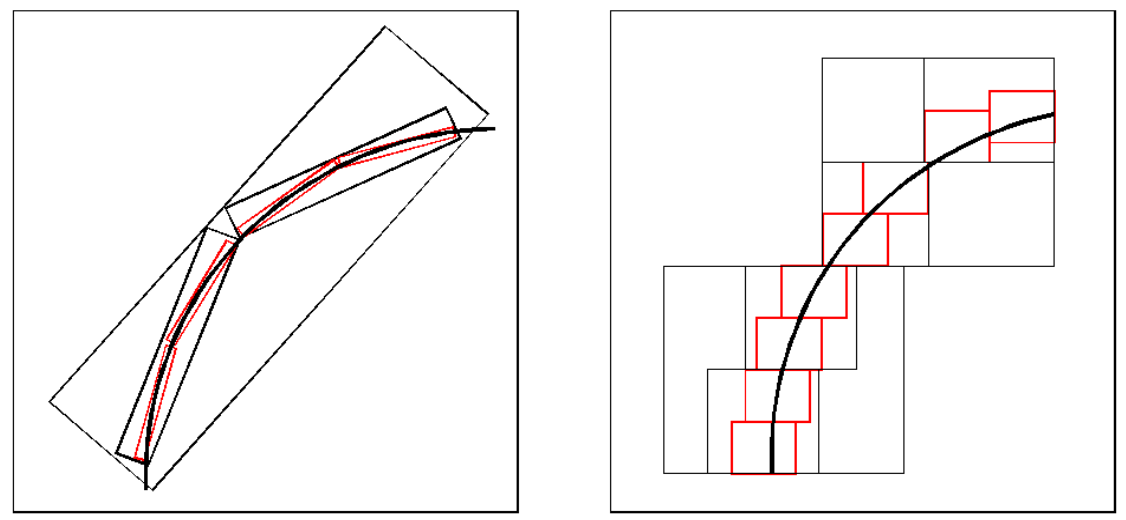

Figure 5 Representation of curved singularities using wavelets (right) and curvelets (left) [5].

\section{Data and Method}

\subsection{Area of study}

The study area is a residential area in Ismailia city about $120 \mathrm{KMs}$ to the north east direction from Cairo the capital of EGYPT. The study area is an urban area comprises scattered buildings, roads, vegetation areas and shadowed areas. The data was provided by Digital Globe, http://www.digitalglobe.com. The images were captured on April 7th, 2011 in morning time. Figure 9 illustrates false-color composite, NIR-2 G B of part of the study area. 


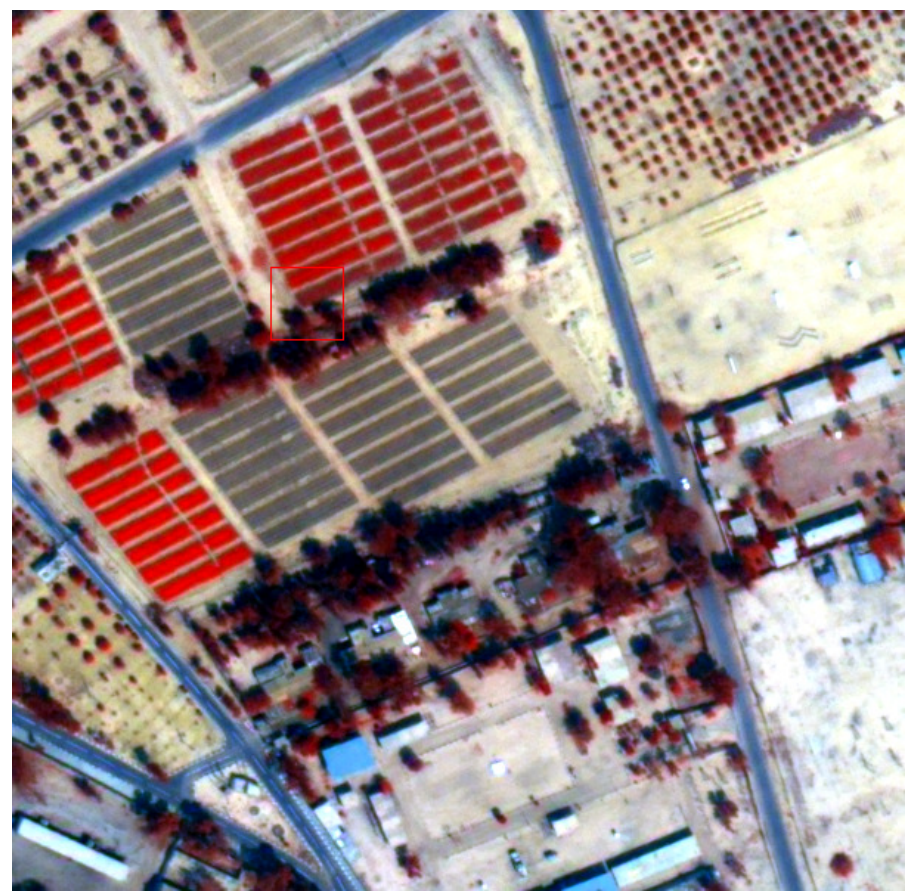

Figure 6. Area of study

\subsection{Methodology}

Basic spectral information for mapping applications such as land-use surveys are essentially provided by the multispectral bands. However, as the limitation to the data-storage volume and transmitting capability of the satellite, satellites do not collect high-resolution multispectral images directly. So, what happen to be the sensor collects only one panchromatic band, wide range of the spectrum, with higher spatial resolution and the rest of the bands, with narrower ranges of the spectrum, with lower resolution[12]. In case of WorldView-2 the panchromatic band volume is twice as the whole 8 -spectral bands together. Considering these limitations, clearly that effective image fusion techniques are the most effective solution for providing high-spatial-resolution and high-spectral-resolution remote sensing images.

The proposed algorithm begins with a data fusion between the panchromatic band of the Worldview data, $0.50 \mathrm{~m}$, and the multispectral ones, $2.00 \mathrm{~m}$ resolution, to generate 8 -spectral bands with a resolution of $0.50 \mathrm{~m}$. One of the most common fusion techniques is the Brovey Transform. This technique is optimum when an increase in contrast in the low and high ends of an images histogram (i.e., to provide contrast in shadows, water, and high reflectance areas such as urban features) is needed. The procedure of this transforms starts with multiplying each MS band by the high-resolution PAN band, and then divides each product by the sum of the MS bands. Since the Brovey Transform is intended to produce RGB images, only three bands at a time should be merged from the input multispectral scene[12] in our case NIR-2, Green and Blue bands were chosen.

Based on the curvelet transform theory a new implementation for detecting edges will be introduced depending on the fact that the values of curvelet coefficients are determined by how they are aligned in the real image, the more accurately a curvelet is aligned with a given curve in an image; the higher is its coefficient value. Analyzing these coefficients, it can be found that the coefficient in each scale level contains different information. Consequently, by arranging the coefficients of each level and take the most significant part of them, this will enhance the 
edge information that represents the important part of the image to us. Then, the coefficients are reconstructed to get a new image where the edge parts are enhanced. Morphological filters will be applied to remove the undesired noisy pixels. Figure 7 represents a schematic diagram of the aforementioned algorithm.

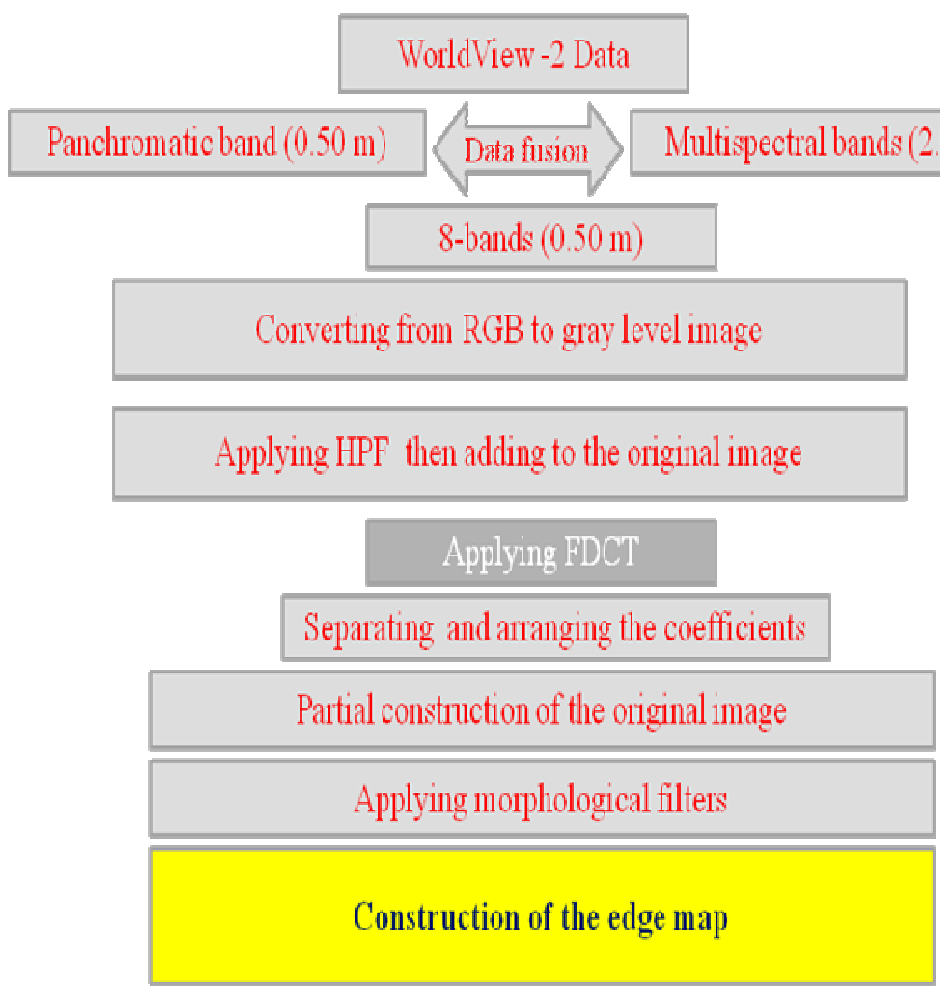

Figure 7. The proposed algorithm

\section{Results and discussion}

The proposed algorithm is mainly depending on the curvelet transform with pre-processing steps. The pre-processing steps involve data fusion between the multispectral bands with 2.00 $\mathrm{m}$ resolution with the panchromatic band with $0.5 \mathrm{~m}$ resolution. The resulting image will be a multi spectral image with 8-bands. The next step is to convert the RGB image to gray level image using the standard perceptual weightings for the three-color components RGB using the following equation (http://www.mathworks.com/help/toolbox/wavelet/gs/f4-1013594.html).

$$
\text { Gray level }=0.2990 \times R+0.5870 \times G+0.1140 \times B \quad \text { Eq.4 }
$$

After that, a high-pass filter is applied, the resulting image will be added to the original image as in Figure 8. 
International Journal of Computer Science \& Engineering Survey (IJCSES) Vol.3, No.4, August 2012

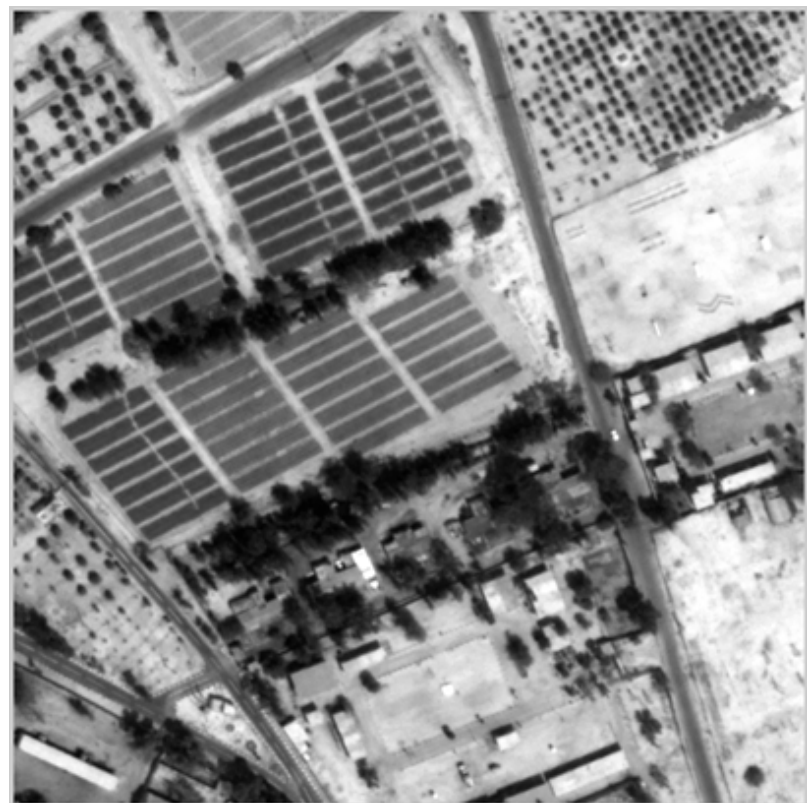

Figure 8 . The grey level image after high pass results added to the original image

The 2D FFT of the output image is then calculated to obtain Fourier's samples. And according to the size of the original image, the scale levels are determined by using

$$
n=\log _{2}(N)-3, \quad \text { Eq. } 5
$$

Where $N$ is the minimum number of the image size and $n$ is the number of the scale levels, i.e. for $N=512$ pixels, the $n=6$ levels. These scale levels are divided into three parts, which are coarse level, detail level and fine level. Then curvelet transform is applied to extract the coefficients from these parts. Images are then reconstructed for each level with those coefficients as shown in Figure 9.
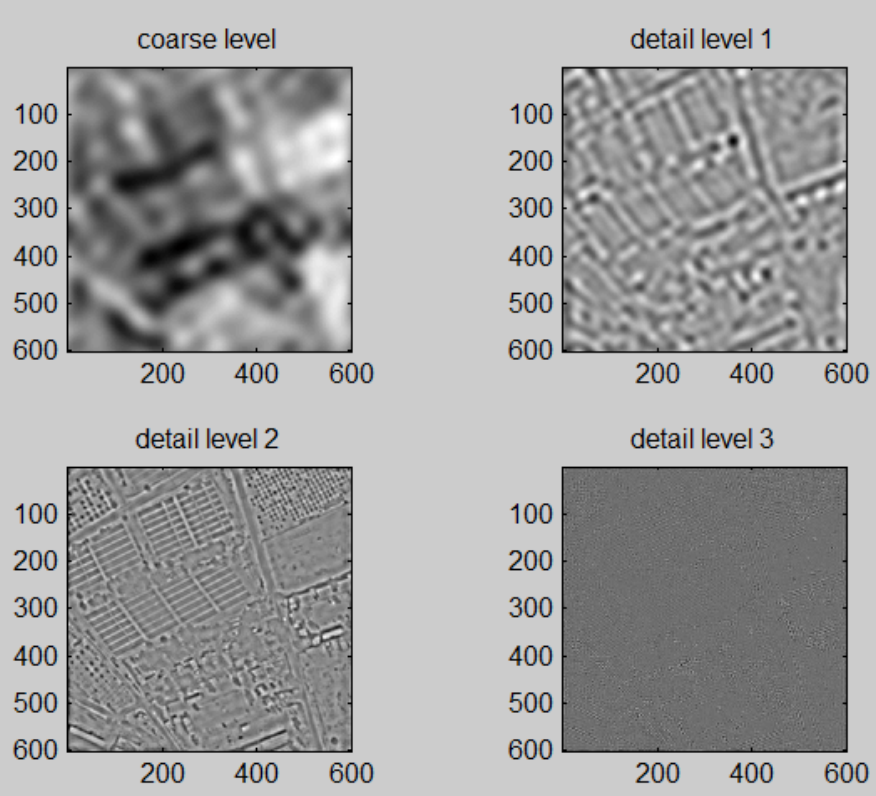

Figure 9. The reconstructed images using different scales levels 
Analyzing the curvelet transform coefficients, it can be found that they contain different information in each scale level. Consequently, by arranging the coefficients of each level and take the most significant part of them, this will enhance the edge information that represents the image part of interest. Then, the coefficients are reconstructed to get a new image called the edge map, as shown in Figure 10, where the edge parts are enhanced.

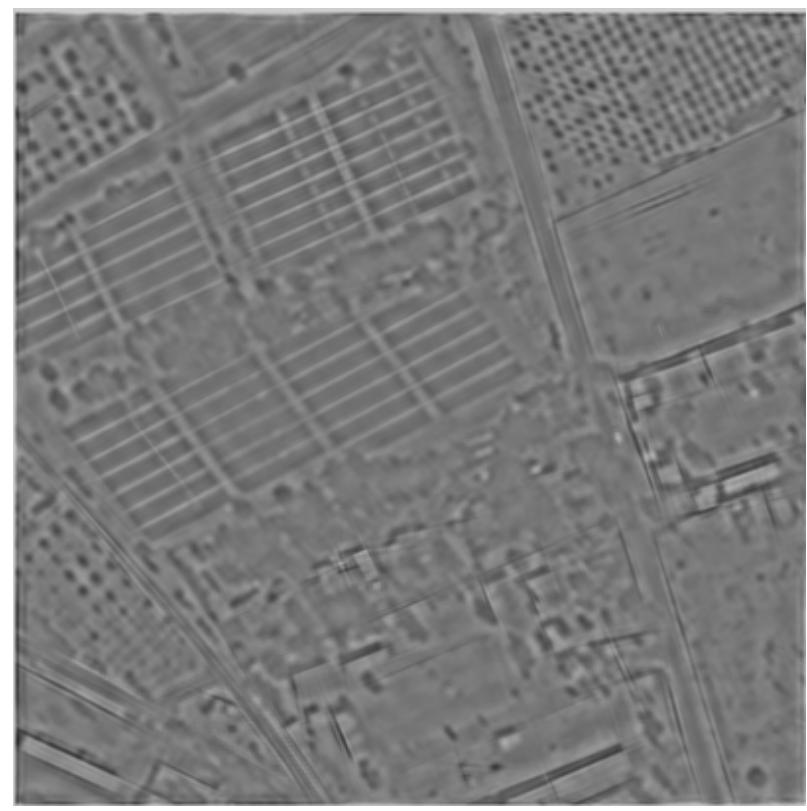

Figure 10. The reconstructed edge map

Table 1 summarizes the total number of coefficient in each scale and the actual used percentage.

\begin{tabular}{|c|c|c|c|}
\hline Scale & $\begin{array}{c}\text { No. of total } \\
\text { coefficients }\end{array}$ & $\begin{array}{c}\text { Percentage } \\
\text { used }\end{array}$ & $\begin{array}{c}\text { No. of used } \\
\text { coefficients }\end{array}$ \\
\hline 1 & 625 & $0 \%$ & 0 \\
\hline 2 & 8320 & $0 \%$ & 0 \\
\hline 3 & 32032 & $100 \%$ & 32032 \\
\hline 4 & 124064 & $100 \%$ & 124064 \\
\hline 5 & 491264 & $1 \%$ & 4912 \\
\hline 6 & 1944856 & $1 \%$ & 19448 \\
\hline Total & 2601161 & $6.9 \%$ & 180456 \\
\hline
\end{tabular}

Table 1. The percentage used in reconstructing the edge map image

The edge map is then thresholded to get enhanced edge map as in Figure 11. The reconstructed edge image was thresholded based on the fact that any edges will have abrupt changes in the pixel from negative towards positive values, and the absolute summation will determine the strong or the weakness of that particular edge. 
International Journal of Computer Science \& Engineering Survey (IJCSES) Vol.3, No.4, August 2012

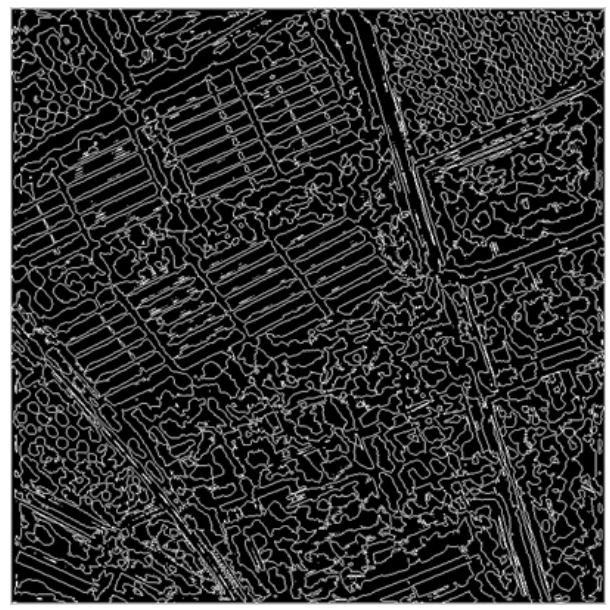

Figure 11. The thresholded edge map

Figure 12 demonstrates the criteria of thresholding, two points on both sides of a strong edge with indices' values +5.503 and -5.43 respectively, while the same figure (down) illustrates two points on the sides of a weak edge having the values of -26.35 and -21 respectively, this is why first point will be considered as an edge while second point is not.
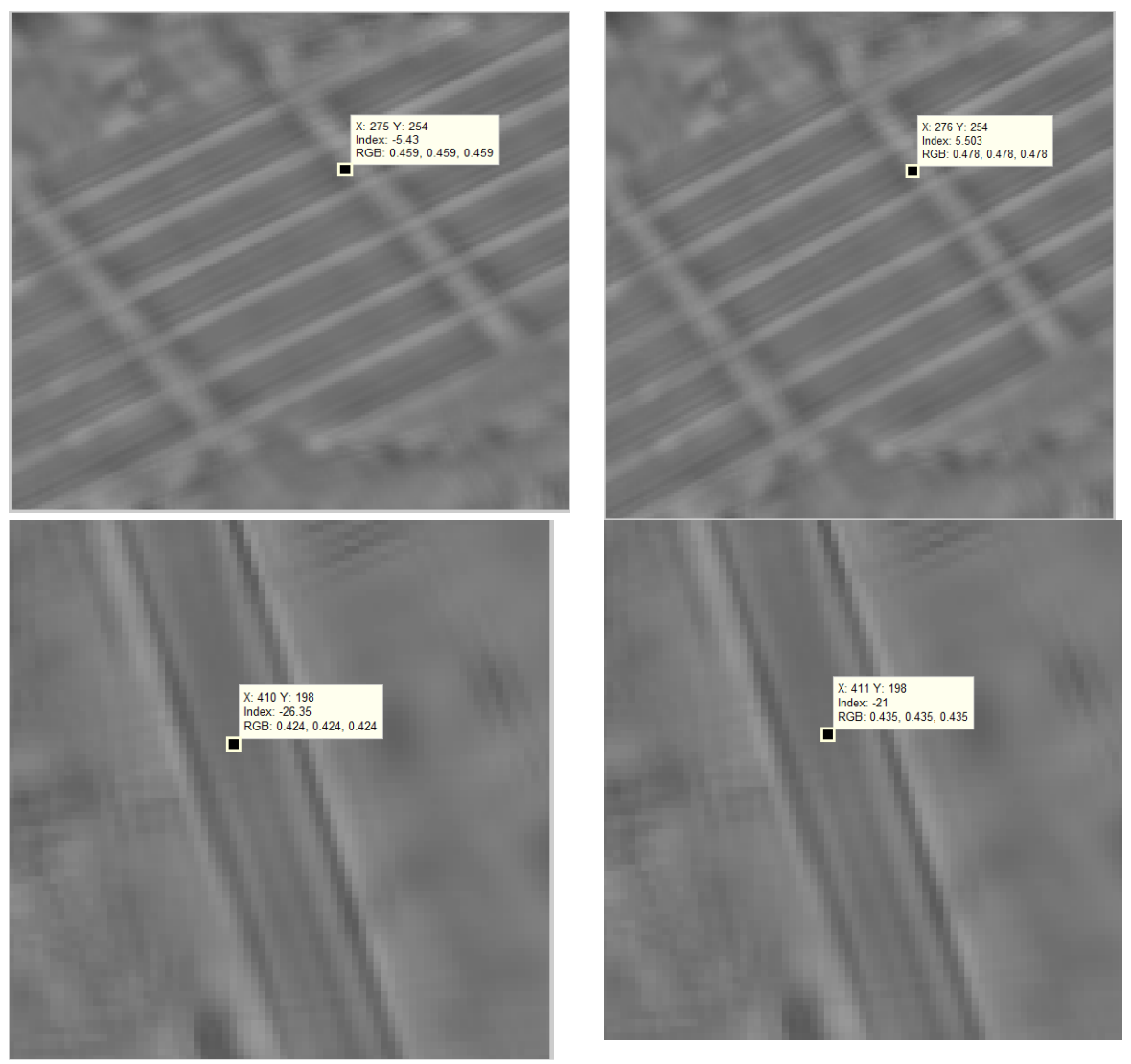

Figure 12. An example of the thresholding criteria

The next step is applying morphological filters to get rid of undesired artifacts, i.e. isolated pixels below certain threshold, and the result is in Figure 13. 
International Journal of Computer Science \& Engineering Survey (IJCSES) Vol.3, No.4, August 2012

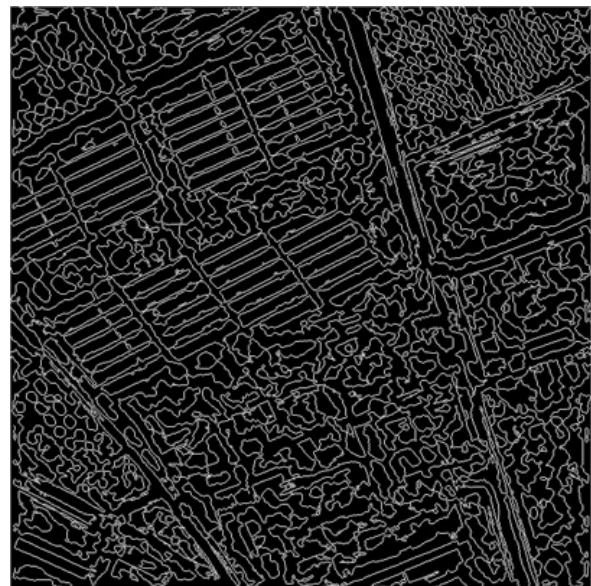

Figure 13. Final edge map after morphological filters

To illustrate the quality of this algorithm compared with Canny and wavelet transforms, the procedure was repeated using these two methods and the final result is in Figures 14 and 15. Canny was used with sigma equal to 1 and the thresholds $\mathrm{T} 1=0.006$ and $\mathrm{T} 2=0.02$.

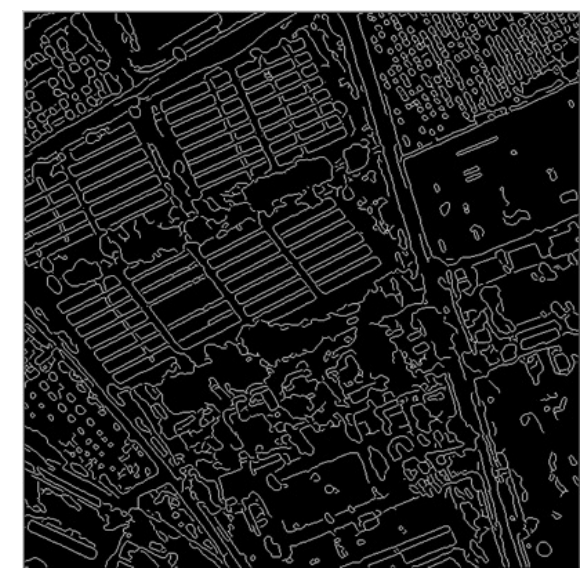

Figure 14 results with Canny Operator

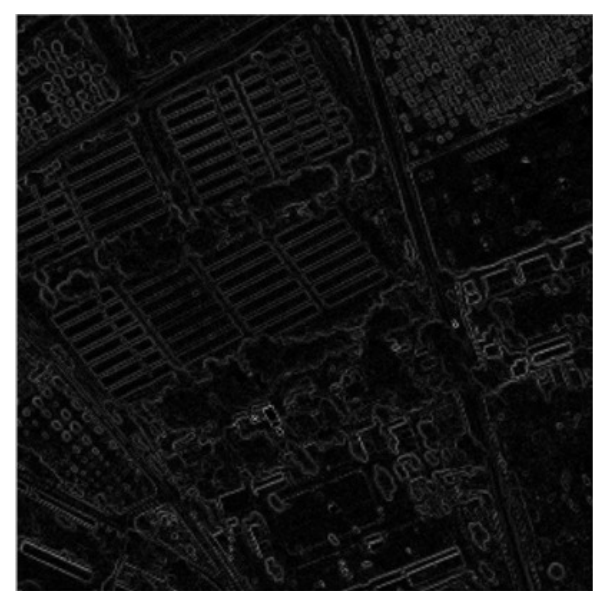

Figure 15 results with Wavelet transforms 
The result of Canny show almost identical result with the curvelet transforms edge detection result. The case was different with the wavelet as in the Figure 15, which illustrates the edge detection result when using the original image as an input to the wavelet transforms.
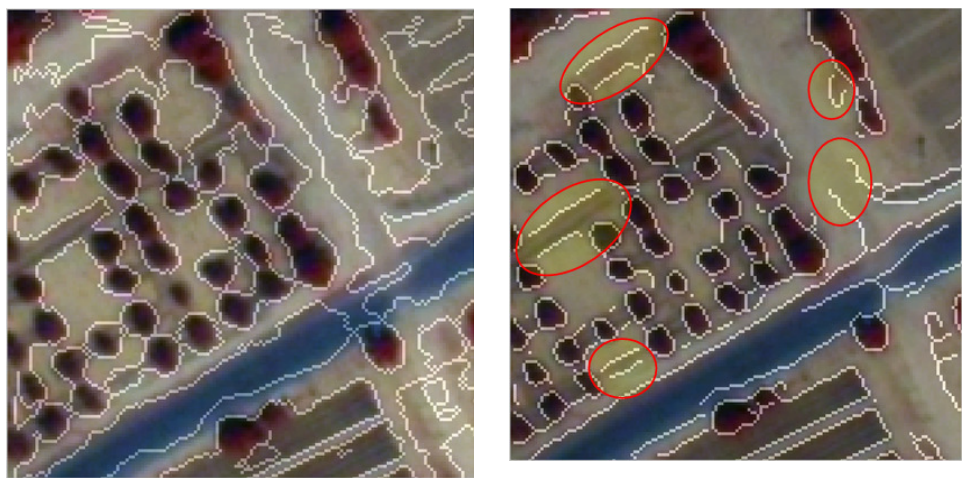

Figure 16 highlighting a certain area in curvelet and Canny

A tiny area in the original image, figure 8 , was picked and enlarged to emphasize the potentials of using the curvelet transform as an edge detector against Canny and wavelet transform. Figure 16 is highlighting a small area in both Curvelet (left) and Canny edge map (right) overlaid on the original image. It was very clear that Canny wasn't able to siege or block many features leaving a lot of open boundaries, while curvelet gave the most accurate delineation of the edges better than the Canny.

\section{Conclusions}

The proposed algorithm was applied with curvelet transform on a high-resolution satellite imagery data, and repeated using wavelet and Canny operators, the results were promising. The total number of coefficients used to reconstruct the edge map using curvelet transforms was 180456 , representing $6.9 \%$ of the total coefficients, while in case of wavelet, the coefficients' number was $360000,100 \%$ of the total coefficients.

Curvelet transforms give close or even improved delineation to edges compared to Canny and a far better than the wavelet transforms. This method can be used as an important layer for a further classification step as it gives a closed boundary for almost all features in the input image. Although Curvelet transforms is promising and efficient for edge detection, there is one drawback must be regarded in the future, which one is that the quality of the edge detection is a function of the pre-processing steps (the high-pass filter to enhance edges), as any edge detector will suffer from the great deal of heterogeneity of the images, especially when using very high resolution imagery, which will be the motivation for more distant investigation in the near future.

\section{Acknwledgments}

This work was supported in part by research funds from TECTERRA Commercialization and Research Centre, the Canada Research Chairs Program, and the Natural Science and Engineering Research Council of Canada (NSERC).

\section{References}

[1] L. Zhenghai and Q. Jianxiong, "Study of Technique of Edge Detection Based on Curvelet Transform," in Second International Symposium on Computational Intelligence and Design, 2009, pp. 543-545.

[2] Y. O. Ouma, et al., "Urban features recognition and extraction from very-high resolution multi-spectral satellite imagery: a micro-macro texture determination and integration framework," IET Image Processing, vol. 4, p. 235, 2010. 
International Journal of Computer Science \& Engineering Survey (IJCSES) Vol.3, No.4, August 2012

[3] E. Candes, et al., "Fast discrete curvelet transforms," Multiscale Modeling \& Simulation, vol. 5, pp. 861899, 2006.

[4] T. Geback and P. Koumoutsakos, "Edge detection in microscopy images using curvelets," BMC Bioinformatics, vol. 10, p. 75, 2009.

[5] T. Guha and Q. M. J. Wu, "Curvelet Based Feature Extraction," in Face Recognition, M. Oravec, Ed., ed: InTech, Available from: http://www.intechopen.com/articles/show/title/curvelet-based-feature-extraction, 2010, pp. 35-46.

[6] M. Xiao, et al., "Edge Detection of Riverway in Remote Sensing Images Based on Curvelet Transform and GVF Snake," presented at the Spatial Accuracy Assessment in Natural Resources and Environmental Sciences, Shanghai, P. R. China, , 2008.

[7] J. Canny, "A Computational Approach to Edge Detection," Pattern Analysis and Machine Intelligence, IEEE Transactions on, vol. PAMI-8, pp. 679-698, 1986.

[8] S. Livens, et al., "Wavelets for texture analysis, an overview," in Image Processing and Its Applications, 1997., Sixth International Conference on, 1997, pp. 581-585 vol.2.

[9] S. G. Mallat, "A theory for multiresolution signal decomposition: the wavelet representation," Pattern Analysis and Machine Intelligence, IEEE Transactions on, vol. 11, pp. 674-693, 1989.

[10] D. L. Donoho and M. R. Duncan, "Digital curvelet transform: Strategy, implementation and experiments," in Wavelet Applications Vii. vol. 4056, H. H. Szu, et al., Eds., ed, 2000, pp. 12-30.

[11] J. Ma and G. Plonka, "Computing with Curvelets: From Image Processing to Turbulent Flows," Computing in Science and Engg., vol. 11, pp. 72-80, 2009.

[12] K .G. Nikolakopoulos, "Comparison of nine fusion techniques for very high resolution data," Photogrammetric Engineering and Remote Sensing, vol. 74, pp. 647-659, May 2008. 